\title{
Growth Characteristics Modeling of Mixed Culture of Bifidobacterium bifidum and Lactobacillus acidophilus using Response Surface Methodology and Artificial Neural Network
}

\author{
Ganga Sahay Meena ${ }^{1 *}$, Gautam Chandra Majumdar ${ }^{1}$, Rintu Banerjee ${ }^{1}$, Nitin Kumar ${ }^{1}$ and \\ Pankaj Kumar Meena ${ }^{2}$ \\ ${ }^{1}$ Microbial Biotechnology and Downstream Processing Laboratory; Department of Agricultural and Food \\ Engineering; Indian Institute of Technology; Kharagpur - India. ${ }^{2}$ By Product Laboratory; Dairy Technology \\ Division National Dairy Research Institute; Karnal; Haryana - India.
}

\begin{abstract}
Different culture conditions viz. additional carbon and nitrogen content, inoculum size and age, temperature and $\mathrm{pH}$ of the mixed culture of Bifidobacterium bifidum and Lactobacillus acidophilus were optimized using response surface methodology (RSM) and artificial neural network (ANN). Kinetic growth models were fitted for the cultivations using a Fractional Factorial (FF) design experiments for different variables. This novel concept of combining the optimization and modeling presented different optimal conditions for the mixture of B. bifidum and L. acidophilus growth from their one variable at-a-time (OVAT) optimization study. Through these statistical tools, the product yield (cell mass) of the mixture of $\mathrm{B}$. bifidum and $\mathrm{L}$. acidophilus was increased. Regression coefficients $\left(R^{2}\right)$ of both the statistical tools predicted that ANN was better than RSM and the regression equation was solved with the help of genetic algorithms (GA). The normalized percentage mean squared error obtained from the ANN and RSM models were 0.08 and $0.3 \%$, respectively. The optimum conditions for the maximum biomass yield were at temperature $38^{\circ} \mathrm{C}, \mathrm{pH} 6.5$, inoculum volume $1.60 \mathrm{~mL}$, inoculum age $30 \mathrm{~h}$, carbon content $42.31 \%(w / v)$, and nitrogen content $14.20 \%(w / v)$. The results demonstrated a higher prediction accuracy of ANN compared to RSM.
\end{abstract}

Key words: Response surface methodology (RSM), Artificial neural network (ANN), Genetic algorithms (GA), Fractional factorial design (FFD), Bifidobacterium bifidum, Lactobacillus acidophilus

\section{INTRODUCTION}

According to the recently adopted definition by FAO/WHO, probiotics are live microorganisms, which when administered in adequate amounts confer a health benefit on the host (Hemaiswarya et al. 2013). Probiotics are not a new invention but have existed in traditional foods such as beverages, salty fishes, yogurt, different types of cheeses, etc since olden times (Amara and shibl 2013). Several microbial groups have the potential to function as probiotics but the species of
Lactobacillus and Bifidobacterium are the most commonly used as probiotics, including certain yeast and bacilli (Singh et al. 2011). Most bacterial species of this class are formally classified as GRAS (generally recognized as safe) organisms. The popularity of these microbes is based on the millennia of use in food and feed that are used in probiotic dairy drinks and yoghurts since hundreds of years (Sanders 1999). Currently, consumers are very much concerned about the sensorial, nutritional and functional attributes of food worldwide. A number of health benefits, which

*Author for correspondence: gsmndri@gmail.com 
may be direct or indirect such as enhanced barrier function, modulation of the mucosal immune system, production of antimicrobial agents, enhancement of digestion and absorption of food and alteration of the intestinal microflora (Hemaiswarya et al. 2013), anti-mutagenic effects, anti-carcinogenic properties, improvement in lactose metabolism, reduction in serum cholesterol and immune system stimulation (Shah 2007), better calcium absorption, prevention of allergy and reduction in dental decay are associated with the consumption of probiotics (Singh et al. 2011).

According to a recent market research report, 'Probiotics Market (2009;2014)', the global probiotics market generated US $\$ 15.9$ billion in 2008 and was expected to be worth US\$ 32.6 billion by 2014 with a compound annual growth rate of $12.6 \%$ from 2009 to 2014. India is fast emerging as a potential market for probiotics in foods with several companies such as Nestle, Mother Dairy, Danisco, Chr Hansen, Yakult and Danone. The probiotic product industry in India is an estimated ₹ 20.6 million with a projected annual growth rate of $22.6 \%$ until 2015 (Ganguly et al. 2011). Probiotics in India generally come in two forms, viz., milk and fermented milk products with the former constituting a major chunk (50$60 \%$ ) of the market. List of probiotic containing foods is wide and still growing. Main products existing in the market are dairy-based, including fermented milks, cheese, ice cream, buttermilk, milk powder, and yogurts, the latter accounting for the largest share of sales. Usually, dairy products are known as the 'best carrier' of probiotics.

Probiotic bacteria such as Lactobacillus and Bifidobacterium can play an important role in promoting human health in the gastrointestinal tract (Mitsuoka 1990). They actively contribute in the digestion, immune stimulation and inhibition of pathogens such as Bacteroides, Escherichia, Clostriduim and Proteus, which are potentially harmful bacteria found in the gastrointestinal tract (Ziemer and Gibson 1998). Recently, systematic reviews on the health benefits of probiotics in details have been published (Aureli et al. 2011; Amara and Shibl 2013). The effectiveness of the probiotic effects generally depends on the mechanisms by which they exerts their activities. Mostly, to treat a disease, the probiotics follow a set of mechanisms, which were recently reviewed (Hemaiswarya et al. 2013). The primary mechanism for probiotic action is known as competitive colonization, or competitive suppression, best described as the proliferation of probiotic bacteria in the human intestine, leaving little space and food for the growth of any pathogens. Secondary, the by-products (i.e., lactic acid and acetic acid) secreted by these probiotics lowers the $\mathrm{pH}$, thereby creating a hostile environment for the growth of pathogenic microorganisms. The secreted acids also increases the peristalsis, which also helps to speed pathogens out from the intestines (Ballongue 1992; Biavati et al. 2000).

Traditional, i.e., one variable at-a-time (OVAT) method of bacterial growth optimization is not only time consuming but also neglects interactions of different variables, which affects the yield. Process optimization through statistical method is a technique in which changes or adjustments are made in a process to get better results (Myers and Montgomery 2002). There are several techniques for process optimization such as Response Surface Methodology (RSM), Artificial Neural Networks (ANN), Genetic Algorithms (GA), etc. In these engineering applications, a response of interest is usually influenced by several variables and the objective of the engineering applications is to find the variables that can optimize the response. RSM is a tool to study the optimal process parameters that produce a maximum, or minimum value of the response and represents the direct and interactive effects of the process parameters through two and three-dimensional plots (Gangadharan et al. 2008). ANN is computational model of nervous systems. Natural organisms, however, do not possess only nervous systems but also have genetic information stored in the nucleus of their cells (genotype). The nervous system is part of the phenotype, which is derived from this genotype through a process, called development (Rajasekaran and Vijaylakshmi 2004). Using the method of neural networks $(\mathrm{NN})$, the relationship between a set of independent variables $X$ and the dependent variables $Y$ can be obtained. From the given pairs of input $X$ and output $Y$ data, neural network directly learns and develops a relationship between them but does not yield any mathematical equation relating the variables. After the learning, this network is able to predict the correct output from an input data set that has not been previously used during the learning. GA is a tool by which the optimization problems can be accurately solved with in a limited use of computer time (Das 2005). Optimization of various bacterial strains in Erlenmeyer flasks using different optimization 
tools have been reported by several other authors (Nagarjun et al. 2005; Kumari et al. 2009; Lima et al. 2009; Negi and Banerjee, 2009; Usmiati and Marwati, 2009; Coelho et al. 2011; Meena et al. 2011; Meena et al. 2013).

This study developed the empirical model to increase the cell growth of the mixed culture of $B$. bifidum and $L$. acidophilus (1:1 ratio) by optimizing the growth parameters such as temperature, $\mathrm{pH}$, inoculum volume, incubation period and additional effect of different carbon and nitrogen sources employing RSM, ANN and GA.

\section{MATERIALS AND METHODS}

Micro-organisms and their growth conditions Bifidum bifidum NCDC 255 and L. acidophilus NCDC 14 were obtained in freeze dried state from National Collection of Dairy Cultures (NCDC), National Dairy Research institute, Karnal, Haryana, India. The methods used for the microbial culture activation and pellet extraction was same as earlier reported (Meena et al. 2011; Meena et al. 2013). Composition of the medium used for the growth of mixed culture of $B$. bifidum and $L$. acidophilus $(1: 1)$ contained $(\mathrm{g} / \mathrm{L})$ casein peptone 10 , yeast extract 5 , sodium acetate 5 , Tween $801, \mathrm{MgSO}_{4} 0.2, \mathrm{MnSO}_{4} 0.05, \mathrm{~K}_{2} \mathrm{HPO}_{4} 2$ and its $\mathrm{pH}$ was maintained at 7.0. The growth of the mixed culture was carried out in $250 \mathrm{~mL}$ Erlenmeyer flasks each containing $50 \mathrm{~mL}$ growth medium and maintained at $37{ }^{\circ} \mathrm{C}$. The cell biomass was determined by measuring the optical density (OD) of the medium after $24 \mathrm{~h}$ at $600 \mathrm{~nm}$. Before measuring the $\mathrm{OD}$, the liquid containing cells were centrifuged and washed with sterile distilled water for two times to remove the adhering medium constituents. All the solvents and reagents used in the present study were procured from Merck, Germany.

\section{Experimental design}

\section{Selection of initial parameters}

For the selection of initial parameters, 'one variable at a time method' was used. The different variables, viz. temperature, $\mathrm{pH}$, volume of inoculum, age of inoculum and additional carbon and nitrogen sources were selected for the growth of mixed culture.

\section{Empirical model development using RSM}

In order to find the effect of different growth parameters on the predicted value of bacterial growth $Y_{p}$ was obtained by conducting the experiments on different combination of independent variables (growth parameters), which was obtained from a standard experimental design. During the experiments, the response, or values of dependent variables obtained from each of the combinations of independent variables was measured. A mathematical relationship between the independent and dependent variables was developed. Using this model, the predicted value of response was find out within the domain of limiting values of independent variables. For the different growth parameters, it was desired to develop a polynomial model between the mixed culture growth and growth parameters to develop the following relationship between the coded values of independent variables, i.e., temperature $\left(x_{1}\right), \mathrm{pH}\left(x_{2}\right)$, inoculum volume $\left(x_{3}\right)$, inoculum age $\left(x_{4}\right)$, carbon sources $\left(x_{5}\right)$ and nitrogen sources $\left(x_{6}\right)$ and dependent variable (cell mass of mixed culture, Yp) as shown below. $Y p=b_{o}+b_{1} x_{1}+b_{2} x_{2}+b_{3} x_{3}+b_{4} x_{4}+b_{5} x_{5}+b_{6} x_{6}+b_{7} x_{1}{ }^{2}+b_{8}$
$x_{2}^{2}+b_{9} x_{3}^{2}+b_{10} x_{4}^{2}+b_{11} x_{5}^{2}+b_{12} x_{6}^{2}+b_{13} x_{1} x_{2}+b_{14} x_{1} x_{3}+b$
${ }_{15} x_{1} x_{4}+b_{16} x_{1} x_{5}+b_{17} x_{1} x_{6}+b_{18} x_{2} x_{3}+b_{19} x_{2} x_{4}+b_{20} x_{2} x_{5}+b_{2}$
${ }_{1} x_{2} x_{6}+b_{22} x_{3} x_{4}+b_{23} x_{3} x_{5}+b_{24} x_{3} x_{6}+b_{25} x_{4} x_{5}+b_{26} x_{4} x_{6}+b_{27}$
$x_{5} x_{6}$ (Eq. 1) Where $b_{o}, b_{1,}, b_{2} \ldots \ldots \ldots . .$. etc. are the
regression constants.

\section{Experimental Modeling}

Fractional factorial design

Fractional factorial design is a method by which the numbers of experiments are considerably reduced. This is used for the screening of independent variables, which have large effect on the dependent variables (Das 2005). Using two levels (+1 and -1) factorial design, two values of $l$ and $s$ were found out for all the experimental runs. Here, the values of $l$ and $s$ for two scarifying interactions were $l_{1}, s_{1}$, and $l_{2}, s_{2}$, respectively. With the help of factorial design, different $\mathrm{s}$ values were identified as $\left(s_{1}=0, s_{2}=0\right),\left(s_{1}=0, s_{2}=1\right),\left(s_{1}=\right.$ $\left.1, s_{2}=0\right)$, and $\left(s_{1}=1, s_{2}=1\right)$. All the experiments were conducted according to $s_{1}=0$ and $s_{2}=0$ design (Meena et al. 2011), during present investigation.

\section{Optimization}

\section{Artificial neural network modeling}

In this present investigation, a feed forward back propagation neural network (Meena et al. 2011; Meena et al. 2013) was used to evaluate its capability in cell mass yield prediction of mixed culture. In this process, ANN computed the error 
between the desired (predicted) response and the actual (experimental) response. The number of neurons in input layer, hidden layer and output layer of this neural network were kept as 6,11 and 1, respectively. This ANN was first trained with reported data of B. bifidum (Meena et al. 2011). After training, it was able to predict the cell mass yield of the mixed culture accurately through error minimization that was compared with the predicted value of cell mass yield obtained from RSM (Meena et al. 2013).

\section{Genetic Algorithms}

In order to maximize the cell mass yield of the mixed culture, GA was applied to the developed ANN based model (Meena et al. 2011; Meena et al. 2013) by monitoring above mentioned six growth parameters. It was posed as the minimization of problem associated with the optimization studies. Genetic optimization was continued till the maximum cell mass yield obtained.

\section{Software used}

For the proper execution of ANN and GA, MATLAB 7.0 was used to develop the empirical model.

\section{RESULTS AND DISCUSSION}

\section{Selection of initial parameters}

Different growth variables for mixed culture growth were selected by OVAT method and results showed in Figure 1 (A - F). All these parameters, their variation and optimum values are given in Table 1.

Table 1 - Values of different parameters for single parameter optimization.

\begin{tabular}{llc}
\hline Different growth parameters & \multicolumn{1}{c}{ Variation of parameters } & Maximum growth on parameter \\
\hline Temperature, $\left({ }^{\circ} \mathrm{C}\right)$ & $30,35,37,40,45$ & 37 \\
$\mathrm{pH}$ & $4.0,4.5,5.0,5.5,6.0,6.5,7.0,7.58 .0$ & 7.0 \\
Inoculum Volume, $(\mathrm{mL})$ & $0.5,1.0,1.5,2.0,2.5,3.0,3.5$ & 24 \\
Inoculum age, $(\mathrm{h})$ & $12,24,36,48$ & Lactose \\
Carbon sources, $(\% \mathrm{w} / \mathrm{v})$ & Glucose, Fructose, Sucrose Lactose, Xylose & Potassium nitrate \\
Nitrogen sources, $(\% \mathrm{w} / \mathrm{v})$ & Sodium nitrate, Urea, Leucine, Glycine, & \\
& Potassium nitrate, Ammonium sulphate, & \\
& Ammonium chloride, Ammonium nitrate & \\
\hline
\end{tabular}


Figure 1 - Selection of different parameters for mixed culture growth (A. Selection of initial temperature, B. Selection of initial pH, C. Selection of initial inoculun volume, D. Selection of initial incubation period, E. Selection of suitable carbon source and F. Selection of suitable nitrogen source). 


\section{Empirical model development}

From the initial parameter selection, the maximum and minimum values of six independent parameters for mixed culture were fixed as shown in Table 2. Then, a model was developed between the coded values of independent variables $\left(x_{1}, x_{2}\right.$, $x_{3}, x_{4}, x_{5}$ and $\left.x_{6}\right)$ and dependent variable $\left(Y_{p}\right)$ by conducting the experiments according to Fractional Factorial design. All these combinations are given in Table 3 with their corresponding $l$ and $s$ values. Various combination of process variable found at $s_{1}=0, s_{2}=0$ is shown in the Table 4 with their experimental value $(Y e)$ for growth of mixed culture.

Table 2 - Limiting value of independent variables.

\begin{tabular}{lcc}
\hline Parameters & $\begin{array}{c}\text { Maximum } \\
\text { value }\end{array}$ & $\begin{array}{c}\text { Minimum } \\
\text { value }\end{array}$ \\
\hline Temperature, $\left({ }^{\circ} \mathrm{C}\right)$ & 45 & 30 \\
$\mathrm{pH}$ & 8.0 & 4.0 \\
Inoculum volume, $(\mathrm{mL})$ & 3.5 & 0.5 \\
Inoculum age, $(\mathrm{h})$ & 48 & 12 \\
Carbon content, $(\% \mathrm{w} / \mathrm{v})$ & 42.06 & 30 \\
Nitrogen content, $(\% \mathrm{w} / \mathrm{v})$ & 46.67 & 14
\end{tabular}

Table 3 - Values of $l$ and $s$ for various experimental runs with 6 independent variables using $x_{1} x_{2} x_{3} x_{4} x_{5}$ and $x_{2} x_{3} x_{4} x_{5} x_{6}$ as sacrificing interactions.

\begin{tabular}{|c|c|c|c|c|c|c|c|c|}
\hline \multirow{2}{*}{$\begin{array}{c}\text { Experiment } \\
\text { No. }\end{array}$} & \multicolumn{6}{|c|}{ Coded values of independent variables } & \multicolumn{2}{|c|}{ Sacrificing interactions } \\
\hline & $x_{1}$ & $x_{2}$ & $x_{3}$ & $x_{4}$ & $x_{5}$ & $x_{6}$ & $x_{1} x_{2} x_{3} x_{4} x_{5}\left(l_{1}, s_{1}\right)$ & $x_{2} x_{3} x_{4} x_{5} x_{6}\left(l_{2}, s_{2}\right)$ \\
\hline 1 & 1 & 1 & 1 & 1 & 1 & 1 & 5,1 & 5,1 \\
\hline 2 & 1 & 1 & 1 & 1 & 1 & -1 & 5,1 & 4,0 \\
\hline 3 & 1 & 1 & 1 & 1 & -1 & 1 & 4,0 & 4,0 \\
\hline 4 & 1 & 1 & 1 & 1 & -1 & -1 & 4,0 & 3,1 \\
\hline 5 & 1 & 1 & 1 & -1 & 1 & 1 & 4,0 & 4,0 \\
\hline 6 & 1 & 1 & 1 & -1 & 1 & -1 & 4,0 & 3,1 \\
\hline 7 & 1 & 1 & 1 & -1 & -1 & 1 & 3,1 & 3,1 \\
\hline 8 & 1 & 1 & 1 & -1 & -1 & -1 & 3,1 & 2,0 \\
\hline 9 & 1 & 1 & -1 & 1 & 1 & 1 & 4,0 & 4,0 \\
\hline 10 & 1 & 1 & -1 & 1 & 1 & -1 & 4,0 & 3,1 \\
\hline 11 & 1 & 1 & -1 & 1 & -1 & 1 & 3,1 & 3,1 \\
\hline 12 & 1 & 1 & -1 & 1 & -1 & -1 & 3,1 & 2,0 \\
\hline 13 & 1 & 1 & -1 & -1 & 1 & 1 & 3,1 & 3,1 \\
\hline 14 & 1 & 1 & -1 & -1 & 1 & -1 & 3,1 & 2,0 \\
\hline 15 & 1 & 1 & -1 & -1 & -1 & 1 & 2,0 & 2,0 \\
\hline 16 & 1 & 1 & -1 & -1 & -1 & -1 & 2,0 & 1,1 \\
\hline 17 & 1 & -1 & 1 & 1 & 1 & 1 & 4,0 & 4,0 \\
\hline 18 & 1 & -1 & 1 & 1 & 1 & -1 & 4,0 & 3,1 \\
\hline 19 & 1 & -1 & 1 & 1 & -1 & 1 & 3,1 & 3,1 \\
\hline 20 & 1 & -1 & 1 & 1 & -1 & -1 & 3,1 & 2,0 \\
\hline 21 & 1 & -1 & 1 & -1 & 1 & 1 & 3,1 & 3,1 \\
\hline 22 & 1 & -1 & 1 & -1 & 1 & -1 & 3,1 & 2,0 \\
\hline 23 & 1 & -1 & 1 & -1 & -1 & 1 & 2,0 & 2,0 \\
\hline 24 & 1 & -1 & 1 & -1 & -1 & -1 & 2,0 & 1,1 \\
\hline 25 & 1 & -1 & -1 & 1 & 1 & 1 & 3,1 & 3,1 \\
\hline 26 & 1 & -1 & -1 & 1 & 1 & -1 & 3,1 & 2,0 \\
\hline 27 & 1 & -1 & -1 & 1 & -1 & 1 & 2,0 & 2,0 \\
\hline 28 & 1 & -1 & -1 & 1 & -1 & -1 & 2,0 & 1,1 \\
\hline 29 & 1 & -1 & -1 & -1 & 1 & 1 & 2,0 & 2,0 \\
\hline 30 & 1 & -1 & -1 & -1 & 1 & -1 & 2,0 & 1,1 \\
\hline 31 & 1 & -1 & -1 & -1 & -1 & 1 & 1,1 & 1,1 \\
\hline 32 & 1 & -1 & -1 & -1 & -1 & -1 & 1,1 & 0,0 \\
\hline 33 & -1 & 1 & 1 & 1 & 1 & 1 & 4,0 & 5,1 \\
\hline 34 & -1 & 1 & 1 & 1 & 1 & -1 & 4,0 & 4,0 \\
\hline 35 & -1 & 1 & 1 & 1 & -1 & 1 & 3,1 & 4,0 \\
\hline 36 & -1 & 1 & 1 & 1 & -1 & -1 & 3,1 & 3,1 \\
\hline 37 & -1 & 1 & 1 & -1 & 1 & 1 & 3,1 & 4,0 \\
\hline 38 & -1 & 1 & 1 & -1 & 1 & -1 & 3,1 & 3,1 \\
\hline 39 & -1 & 1 & 1 & -1 & -1 & 1 & 2,0 & 3,1 \\
\hline 40 & -1 & 1 & 1 & -1 & -1 & -1 & 2,0 & 2,0 \\
\hline 41 & -1 & 1 & -1 & 1 & 1 & 1 & 3,1 & 4,0 \\
\hline 42 & -1 & 1 & -1 & 1 & 1 & -1 & 3,1 & 3,1 \\
\hline 43 & -1 & 1 & -1 & 1 & -1 & 1 & 2,0 & 3,1 \\
\hline 44 & -1 & 1 & -1 & 1 & -1 & -1 & 2,0 & 2,0 \\
\hline 45 & -1 & 1 & -1 & -1 & 1 & 1 & 2,0 & 3,1 \\
\hline
\end{tabular}


(Cont. Table 3)

\begin{tabular}{|c|c|c|c|c|c|c|c|c|}
\hline \multirow{2}{*}{$\begin{array}{c}\text { Experiment } \\
\text { No. }\end{array}$} & \multicolumn{6}{|c|}{ Coded values of independent variables } & \multicolumn{2}{|c|}{ Sacrificing interactions } \\
\hline & $x_{1}$ & $x_{2}$ & $x_{3}$ & $x_{4}$ & $x_{5}$ & $x_{6}$ & $x_{1} x_{2} x_{3} x_{4} x_{5}\left(l_{1}, s_{1}\right)$ & $x_{2} x_{3} x_{4} x_{5} x_{6}\left(l_{2}, s_{2}\right)$ \\
\hline 46 & -1 & 1 & -1 & -1 & 1 & -1 & 2,0 & 2,0 \\
\hline 47 & -1 & 1 & -1 & -1 & -1 & 1 & 1,1 & 2,0 \\
\hline 48 & -1 & 1 & -1 & -1 & -1 & -1 & 1,1 & 1,1 \\
\hline 49 & -1 & -1 & 1 & 1 & 1 & 1 & 3,1 & 1,1 \\
\hline 50 & -1 & -1 & 1 & 1 & 1 & -1 & 3,1 & 3,1 \\
\hline 51 & -1 & -1 & 1 & 1 & -1 & 1 & 2,0 & 3,1 \\
\hline 52 & -1 & -1 & 1 & 1 & -1 & -1 & 2,0 & 2,0 \\
\hline 53 & -1 & -1 & 1 & -1 & 1 & 1 & 2,0 & 3,1 \\
\hline 54 & -1 & -1 & 1 & -1 & 1 & -1 & 2,0 & 2,0 \\
\hline 55 & -1 & -1 & 1 & -1 & -1 & 1 & 1,1 & 2,0 \\
\hline 56 & -1 & -1 & 1 & -1 & -1 & -1 & 1,1 & 1,1 \\
\hline 57 & -1 & -1 & -1 & 1 & 1 & 1 & 2,0 & 3,1 \\
\hline 58 & -1 & -1 & -1 & 1 & 1 & -1 & 2,0 & 2,0 \\
\hline 59 & -1 & -1 & -1 & 1 & -1 & 1 & 1,1 & 2,0 \\
\hline 60 & -1 & -1 & -1 & 1 & -1 & -1 & 1,1 & 1,1 \\
\hline 61 & -1 & -1 & -1 & -1 & 1 & 1 & 1,1 & 2,0 \\
\hline 62 & -1 & -1 & -1 & -1 & 1 & -1 & 1,1 & 1,1 \\
\hline 63 & -1 & -1 & -1 & -1 & -1 & 1 & 0,0 & 1,1 \\
\hline 64 & -1 & -1 & -1 & -1 & -1 & -1 & 0,0 & 0,0 \\
\hline
\end{tabular}

Table 4 - Experimental design for mixed culture of B. bifidum and L. acidophilus growth with experimental (Ye), RSM and ANN predicted values of mixed culture cell biomass.

\begin{tabular}{|c|c|c|c|c|c|c|c|c|c|}
\hline $\begin{array}{l}\text { Run } \\
\text { order }\end{array}$ & $\begin{array}{l}\text { Temp. } \\
{ }^{0} \mathrm{C}\left(x_{1}\right)\end{array}$ & $\begin{array}{l}\mathrm{pH} \\
\left(x_{2}\right)\end{array}$ & $\begin{array}{l}\text { Inoculum } \\
\text { volume } \\
(\mathrm{mL})\left(x_{3}\right)\end{array}$ & $\begin{array}{l}\text { Inoculum } \\
\text { age }(h) \\
\left(x_{4}\right)\end{array}$ & $\begin{array}{l}\text { Carbon } \\
\text { content } \\
\% \text { w/v }\left(x_{5}\right)\end{array}$ & $\begin{array}{l}\text { Nitrogen } \\
\text { content } \\
\% \text { w/v }\left(x_{\sigma}\right)\end{array}$ & $\begin{array}{l}\text { Experimental } \\
\text { value }\left(Y_{e}\right) \\
\mathrm{OD}_{600}\end{array}$ & $\begin{array}{l}\text { RSM } \\
\text { predicted } \\
\text { values }\left(Y_{p}\right)\end{array}$ & $\begin{array}{l}\text { ANN } \\
\text { Predicted } \\
\text { values } Y_{p}\end{array}$ \\
\hline 1 & 40.15 & 6.86 & 2.53 & 36.36 & 41.37 & 24.57 & 0.789 & 0.7412 & 0.828 \\
\hline 2 & 40.15 & 6.86 & 2.53 & 23.64 & 40.61 & 24.57 & 0.2 & 0.3088 & 0.222 \\
\hline 3 & 40.15 & 6.86 & 1.47 & 36.36 & 40.61 & 24.57 & 0.136 & 0.3512 & 0.1519 \\
\hline 4 & 40.15 & 6.86 & 1.47 & 23.64 & 41.37 & 24.57 & 0.99 & 0.9081 & 1.022 \\
\hline 5 & 40.15 & 5.63 & 2.53 & 36.36 & 40.61 & 24.57 & 0.345 & 0.4992 & 0.3777 \\
\hline 6 & 40.15 & 5.63 & 2.53 & 23.64 & 41.37 & 24.57 & 0.845 & 0.7069 & 0.883 \\
\hline 7 & 40.15 & 5.63 & 1.47 & 36.36 & 41.37 & 24.57 & 0.642 & 0.6086 & 0.6839 \\
\hline 8 & 40.15 & 5.63 & 1.47 & 23.64 & 40.61 & 24.57 & 0.406 & 0.5267 & 0.443 \\
\hline 9 & 34.85 & 6.86 & 2.53 & 36.36 & 40.61 & 36.11 & 0.746 & 0.85 & 0.787 \\
\hline 10 & 34.85 & 6.86 & 2.53 & 23.64 & 41.37 & 36.11 & 0.985 & 0.7919 & 1.015 \\
\hline 11 & 34.85 & 6.86 & 1.47 & 36.36 & 41.37 & 36.11 & 0.625 & 0.5383 & 0.666 \\
\hline 12 & 34.85 & 6.86 & 1.47 & 23.64 & 40.61 & 36.11 & 0.825 & 0.8949 & 0.863 \\
\hline 13 & 34.85 & 5.63 & 2.53 & 36.36 & 41.37 & 36.11 & 1.12 & 0.9772 & 1.142 \\
\hline 14 & 34.85 & 5.63 & 2.53 & 23.64 & 40.61 & 36.11 & 0.926 & 0.9373 & 0.961 \\
\hline 15 & 34.85 & 5.63 & 1.47 & 36.36 & 40.61 & 36.11 & 0.923 & 1.039 & 0.959 \\
\hline 16 & 34.85 & 5.63 & 1.47 & 23.64 & 41.37 & 36.11 & 1.09 & 0.9137 & 1.114 \\
\hline 17 & 37.5 & 6.25 & 2 & 30 & 40.99 & 30.54 & 0.708 & 0.75 & 0.72 \\
\hline 18 & 37.5 & 6.25 & 2 & 30 & 40.99 & 30.54 & 0.619 & 0.67 & 0.563 \\
\hline 19 & 37.5 & 6.25 & 2 & 30 & 40.99 & 30.54 & 0.758 & 0.8616 & 0.959 \\
\hline 20 & 37.5 & 6.25 & 2 & 30 & 40.99 & 30.54 & 0.682 & 0.8616 & 0.959 \\
\hline 21 & 37.5 & 6.25 & 2 & 30 & 40.99 & 30.54 & 0.912 & 0.8616 & 0.959 \\
\hline 22 & 37.5 & 6.25 & 2 & 30 & 40.99 & 30.54 & 0.912 & 0.8616 & 0.959 \\
\hline 23 & 37.5 & 6.25 & 2 & 30 & 40.99 & 30.54 & 0.98 & 0.8616 & 0.959 \\
\hline 24 & 37.5 & 6.25 & 2 & 30 & 40.99 & 30.54 & 1.07 & 0.8616 & 0.959 \\
\hline 25 & 37.5 & 6.25 & 2 & 30 & 40.99 & 30.54 & 0.995 & 0.8616 & 0.959 \\
\hline 26 & 37.5 & 6.25 & 2 & 30 & 40.99 & 30.54 & 0.98 & 0.8616 & 0.959 \\
\hline 27 & 45 & 6.25 & 2 & 30 & 40.99 & 30.34 & 0.953 & 0.8478 & 0.985 \\
\hline 28 & 30 & 6.25 & 2 & 30 & 40.99 & 30.34 & 0.945 & 1.0502 & 0.979 \\
\hline 29 & 37.5 & 8 & 2 & 30 & 40.99 & 30.34 & 0.001 & 0.067 & 0.003 \\
\hline 30 & 37.5 & 4.5 & 2 & 30 & 40.99 & 30.34 & 0.27 & 0.3013 & 0.296 \\
\hline 31 & 37.5 & 6.25 & 3.5 & 30 & 40.99 & 30.34 & 1 & 1.0507 & 1.03 \\
\hline 32 & 37.5 & 6.25 & 0.5 & 30 & 40.99 & 30.34 & 1.09 & 1.0393 & 1.115 \\
\hline 33 & 37.5 & 6.25 & 2 & 48 & 40.99 & 30.34 & 1.36 & 1.2615 & 1.335 \\
\hline 34 & 37.5 & 6.25 & 2 & 12 & 40.99 & 30.34 & 1.3 & 1.3985 & 1.304 \\
\hline 35 & 37.5 & 6.25 & 2 & 30 & 42.06 & 30.34 & 0.892 & 1.2116 & 0.93 \\
\hline 36 & 37.5 & 6.25 & 2 & 30 & 39.92 & 30.34 & 1.11 & 0.9364 & 1.13 \\
\hline 37 & 37.5 & 6.25 & 2 & 30 & 40.99 & 46.67 & 1.02 & 1.1252 & 1.05 \\
\hline 38 & 37.5 & 6.25 & 2 & 30 & 40.99 & 14 & 0.624 & 0.5189 & 0.664 \\
\hline
\end{tabular}


RSM is mainly used for the optimization of growth conditions, reaction parameter, or scalingup the mixed culture growth conditions (Sen and Babu 2005). Experimental data were fitted to the full quadratic equation and the design matrix and the fitness of each term was analyzed by the means of ANOVA (Kumari et al. 2008). Figure 2 shows the corresponding model coefficients $\left(R^{2} 0.7949\right)$ together with the regression coefficient of determination. This was a measure of how well the regression model could be made to fit the raw data. A self-organizing feature map network was used to

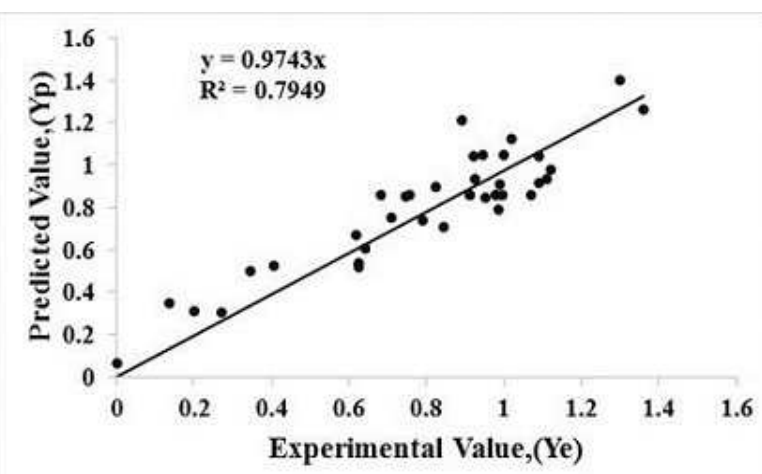

Figure 2 - Determination of regression equation coefficient $\mathrm{R}^{2}$ for mixed culture cell biomass between experimental value (Ye) and RSM predicted values (Yp).

Using MATLAB 7.0, the constants of regression equation and predicted value of dependent variable (OD) were found out. The obtained model for the mixed culture was as given below.

$Y_{p}=0.8578-0.1016 x_{1}-0.1506 x_{2}+0.0056 x_{3}-$ $0.0684 x_{4}+0.1382 x_{5}+0.3032 x_{6}+0.0912 x_{1}^{2}-$ $0.7072 x_{2}^{2}+0.1872 x_{3}^{2}+0.4722 x_{4}^{2}+0.2162 x_{5}^{2}-$ $0.0357 x_{6}^{2}+0.3180 x_{1} x_{2}+0.0676 x_{1} x_{3}-0.2590 x_{1} x_{4}-$ $0.9292 x_{1} x_{5}+1.3050 x_{1} x_{6}-0.0168 x_{2} x_{3}-0.2328 x_{2} x_{4}$ $+0.1855 x_{2} x_{5}-0.0645 x_{2} x_{6}+0.5151 x_{3} x_{4}+$ $0.2316 x_{3} x_{5}+0.2224 x_{3} x_{6}-0.2625 x_{4} x_{5}-.2625 x_{4} x_{6}-$ $1.8158 x_{5} x_{6}$ (Eq. 2)

The predicted value of independent variable and corresponding experimental value for the mixed culture is shown in the Table 5. Genetic algorithms were applied on the data obtained from neural network using MATLAB7.0, which yielded similar results as of ANN but in very short time.

Table 5 showed the optimum value, or combination of different process parameters on predict the growth condition parameters using each independent variable as input layer and growth of mixed culture as response. The least Mean Squared Error (MSE) value and a good prediction of the outputs of both training and validation sets were obtained with four neurons in the hidden layer (Dutta et al. 2004). The $\mathrm{R}^{2}$ value between the actual and estimated responses was determined as 0.9591 (Fig. 3). In ANN modeling, the replicates at center point did not improve the prediction capability of the network because of the similar inputs.

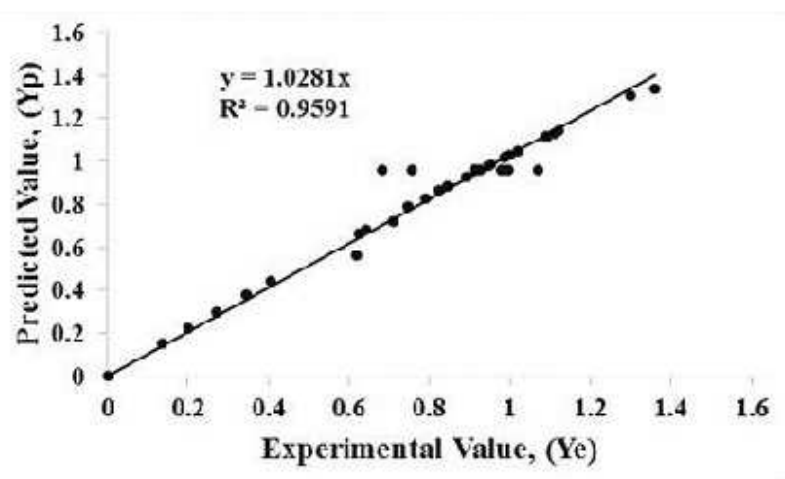

Figure 3 - Determination of regression equation coefficient $\mathrm{R}^{2}$ for the mixed culture cell biomass between experimental value (Ye) and ANN predicted values (Yp).

which the bacterial growth measured by optical density (OD) was highest for the mixed culture.

Table 5 - ANN and GA optimized values of the process parameters for maximum cell biomass of the mixed culture of B. bifidum and L. acidophilus.

\begin{tabular}{ll}
\hline Process parameters & Optimum values \\
\hline Temperature,$\left({ }^{\circ} \mathrm{C}\right)$ & 38 \\
$\mathrm{pH}$ & 6.5 \\
Inoculums volume, $(\mathrm{mL})$ & 1.60 \\
Inoculum age, $(\mathrm{h})$ & 30 \\
Carbon content, $(\%) \mathrm{w} / \mathrm{v}$ & 42.31 \\
Nitrogen content, $(\%) \mathrm{w} / \mathrm{v}$ & 14.20 \\
\hline
\end{tabular}

In the present study, RSM, ANN and GA optimization methodologies were used to predict the growth model of the mixed culture of $B$. bifidum and L. acidophilus (1:1) and optimized the growth parameters. Both the models were capable to predict the combination of independent variables for maximum cell biomass of mixed 
culture but ANN showing more accuracy in estimation.

Regression coefficient $\left(R^{2}\right)$ of ANN and RSM reflected that ANN was better than RSM. RSM was useful in getting insight information (e.g., interactions between different components) of the system directly, but ANN was also equally useful in the sensitivity analysis. ANN showed better modeling technique for data set showing nonlinear relationship over RSM. Thus, ANN could be a very powerful and flexible tool well suited for the development of empirical growth model due to an implicit corrective action arising from the training methodology and the associated estimation procedure. GA optimization results were similar to ANN but delivered within shortest use of computer time as compared to RSM and ANN. Present results showed that the higher cell mass yield of mixed culture was observed at $38^{\circ} \mathrm{C}, \mathrm{pH}$ 6.5 , inoculum volume and age $1.6 \mathrm{~mL}$ and $30 \mathrm{~h}$, respectively, carbon content $42.31 \%(\mathrm{w} / \mathrm{v})$ and nitrogen content $(14.20 \%, \mathrm{w} / \mathrm{v})$. This combination of independent variables could be of significant importance to starter culture producing industries in order to scale- up the production of B. bifidum and $L$. acidophilus on commercial scale more economically due to high cell mass yield.

\section{REFERENCES}

Amara AA, Shibal A. Role of Probiotics in health improvement, infection control and disease treatment and management-review. Saudi Pharmac J. 2013; doi: 10.1016/j.jsps.2013.07.001

Aureli P, Capurso L, Castellazzi AM, Clerici M, Giovannini M, Morelli L. et al. Probiotics and health: An evidence-based review. Pharmacol Res. 2011; 63: 366-376.

Biavati B, Vescovo M, Torriabi S, Bottazzi V. Bifidobacteria: history, ecology, physiology and applications. Analyt Microbiol. 2000; 50: 117-131.

Coelho LF, Lima CJBD, Rodovalho CM, Bernardo MP, Contiero J. Lactic acid production by new Lactobacillus plantarum LMISM6 grown in molasses: optimization of medium composition. Braz J Chem Eng. 2011; 28: 27-36.

Das H. Empirical model development. Hand Book of Food Processing Operations Analysis. $1^{\text {st }}$ ed. New Delhi: Asian Book Private Limited, 2005, 357-382 p.
Dutta JR, Dutta PK, Banerjee R. Optimization of culture parameters for extracellular protease production from a newly isolated Pseudomonas sp. using response surface and artificial neural network models. Process Biochem. 2004; 39: 2193-2198.

Gangadharan D, Sivaramakrishnan S, Nampoothiri KM, Sukumaran RK, Pandey A. Response surface methodology for the optimization of alpha amylase production by Bacillus amyloliquefaciens. Bioresource Technol. 2008; 99: 4597-4602.

Ganguly NK, Bhattacharya SK, Nair GB, Ramakrishna BS, Sachdev HPS, Batish VK. et al. ICMR-DBT guidelines for evaluation of probiotics in food. Indian J Med Res. 2011; 134: 22-25.

Hemaiswarya S, Raja R, Ravikumar R, Carvalho IS. Mechanism of Action of Probiotics. Braz Arch Biol Technol. 2013; 56: 113-119.

Kumari A, Mahapatra P, Banerjee R. Statistical optimization of culture conditions by response surface methodology for synthesis of lipase with Enterobacter aerogenes. Braz Arch Biol Technol. 2009; 52: 1349-1356.

Kumari KS, Babu IS, Rao GH. Process optimization for citric acid production from raw glycerol using response surface methodology, Indian $J$ Biotech. 2008; 7: 496-501.

Lima CJBD, Coelho LF, Blanco KC, Contiero J. Response surface optimization of D (-)-lactic acid production by Lactobacillus SMI8 using corn steep liquor and yeast autolysate as an alternative nitrogen source. Afr J Biotechnol. 2009; 8: 5842-5846.

Meena GS, Gupta S, Majumdar GC, Banerjee R. Growth Characteristics Modeling of Bifidobacterium bifidum Using RSM and ANN. Braz Arch Biol Technol. 2011; 54: 1357-1366.

Meena GS, Kumar N, Majumdar GC, Banerjee R, Meena P K, Yadav V. Growth Characteristics Modeling of Lactobacillus acidophillus Using RSM and ANN. Braz Arch Biol Technol. 2014; 57: 15-22.

Mitsuoka T. Bifidobacteria and their role in human health. J Ind Microbiol. 1990; 6: 263-268.

Myers RM, Montgomery DC. Response Surface Methodology: Process and Product Optimization Using Designed Experiments. $2^{\text {nd }}$ ed. Hoboken: Wiley-Interscience, 2002.

Nagarjun PA, Rao RS, Rajesham S, Rao LV. Optimization of Lactic Acid Production in SSF by Lactobacillus amylovorus NRRL B-4542 Using Taguchi Methodology. J Microbiol. 2005; 43: 38-43.

Negi S, Banerjee R. Optimization of extraction and purification of Glucoamylase produced by Aspergillus awamori in solid state fermentation. Biotechnol Bioproc E. 2009; 14: 60-66. 
Rajasekaran S, Vijaylakshmi PGS. Neural Networks Fuzzy Logic and Genetic Algorithms. $1^{\text {st }}$ ed. New Delhi: Prentice Hall of India, 2004.

Sen R, Babu KS. Modeling and optimization of the process conditions for biomass production and sporulation of a probiotic culture. Process Biochem. 2005; 40: 2531-2538.

Shah NP. Functional Cultures and Health Benefits. Int Dairy J. 2007; 17: 1262-1277.

Singh K, Kallali B, Kumar A, Thaker V. Probiotics: A review. Asian Pac J Trop Biomed. 2011; S287-S290.
Usmiati S, Marwati T. Selection and optimization process of bacteriocin production from Lactobacillus sp. Indones J Agric. 2009; 2: 82-92.

Ziemer CJ, Gibson GR. An overview of Probiotics, Prebiotics and Symbiotic in the Functional Food Concept: Perspectives and Future Strategies. Int Dairy J. 1998; 8: 473-479.

Received: December 12, 2013; Accepted: August12, 2014. 